\title{
Clinical and Experimental Studies in Japanese Encephalitis: Lessons Learnt
}

\author{
UK Misra \\ Department of Neurology, Sanjay Gandhi PGI, Lucknow, India.
}

\begin{abstract}
Introduction: Managing encephalitis for last 3 decades and conducting experimental studies have provided a number of important findings some which are presented.

Method: Summary of important clinical, radiological, neurophysiological and biochemical studies are presented. The results of experimental studies in a rat model have been used to investigate the basis of behavioral changes, histology, and molecular alterations in Japanese encephalitis (JE) and finally an approach to acute encephalitis syndrome (AES) is presented.

Result: The patients with encephalitis can be produced by a number of noninfectious, infectious. The local prevalence and time of the year are crucial for clinical decision making. The affinity of JEV for thalamus, corpus striatum, substantia nigra, cerebellum and anterior horn cell was documented by imaging studies, which was further confirmed on immune histopathological and real time PCR studies highlighting the tropism of JEV. The lower motor neuron involvement on EMG studies was attributed to anterior horn cell involvement. JE results in frequent and severe movement disorders. The basis of movement disorders was revealed in MRI and SPECT studies showing thalamic and cortical hyperperfusion suggesting the involvement of thalamo-cortical projections. Hyperkinetic or hypokinetic movement disorders were due to differential involvement of excitatory or inhibitory circuits in the brain. Reduction in catecholamine and its metabolites in CSF of patients was supported by reduction of catecholamine and dopamine receptors in the JE tropic areas in thalamus, corpus striatum and brainstem resulting in dopamine deficiency. The learning and memory deficits in JE were attributed to cholinergic dysfunction reveled by expression of CHAT, HQNB CHR M2 mscarinic receptors in the JE affected areas of brain.
\end{abstract}

A syndromic approach to AES categorizing the patients into neurologic or systemic group and using rational investigations; imaging and Acyclovir therapy in pure neurologic group and avoiding these in systemic group is recommended. In systemic group, treatment with doxycycline for scrub typhus, artesunate for malaria, ceftriaxone for leptospira and fluid management for dengue are recommended.

Conclusion: A combined clinical and experimental approach provides valuable information to understand the basis of clinical alterations in JE.

Keywords: Japanese Encephalitis, herpes simplex, dengue, chikungunya, syndromic approach, clinical MRI.

Correspondence : Dr. Usha K Misra, Department of Neurology, Sanjay Gandhi PGI, Lucknow, India. Email:drukmisra@rediffmail.com.

DR. BALDEV SINGH ORATION delivered during NAMSCON 2017 at Sri Guru Ram Das Institute of Medical Sciences \& Research, SriAmritsar, Punjab. 


\section{Introduction}

Acute Encephalitis is a global health problem with diverse etiologies. A number of non-infectious conditions (mitochondrial disease, autoimmune encephalitis, cerebral venous sinus thrombosis) and non-viral infection (bacterial or fungal parasitic) may simulate viral encephalitis. Over 100 neurotropic viruses can also result in the syndrome of Acute Encephalitis Syndrome (AES). AES is defined as fever with altered sensorium with or without focal sign of seizures or evidence of raised intracranial pressure after excluding febrile convulsions. The etiology of AES varies according to the geography and season because of difference in the prevalence of organisms and their vectors. Herpes simplex encephalitis (HSE) to the commonest focal encephalitis, which does not have a regional or seasonal preference. Geographically restricted encephalitis is mainly arthropod borne. The commonest endemic viral encephalitis in south east Asia in Japanese encephalitis (JE). West Nile, Chikungunya and Nipah viral encephalitis are also reported from Asia. Murray valley encephalitis, JE and Dengue are reported from Australia; West Nile from middle east, Tick borne encephalitis in Russia and West Nile, St Louise encephalitis have been being reported from America (1). In tropical region, malaria pyogenic meningitis are common and Scrub typhus outbreaks have been reported from tsutsugamishi triangle (2). The clinician has to make a decision at the earliest regarding diagnosis and cost effective treatment. The local prevalence and epidemiology of AES should be kept in mind.

\section{Japanese Encephalitis is the Commonest Cause of Encephalitis in South East Asia}

Japanese encephalitis virus was first isolated from human in Tokyo in 1935. JE virus activity was first detected in India in 1952 by Smithburn et al from Vellore (3). Till $1970 \mathrm{JE}$ was restricted to south India in 1973. JE patients were reported from west Bengal, Bihar, Uttar
Pradesh Assam and Andhra Pradesh (4-6). JE is mainly a disease of rural areas affecting the lower socio economic group. JE has scattered pattern of incidence average there are 1-1.5 patients per village. The ratio of clinical to subclinical cases ranges between 1:10 to 1:1000. JE mainly affects children but in east and north India all age groups are affected suggesting that the virus has been likely introduced in the respective area to relatively non immune population. Occurrence of JE cases has been reported in rainy season, floods, paddy cultivation and pig farming which provide environment for mosquito breeding and transmission of JE virus. Pigs are involved in maintaining and spread of JE virus through a pig, mosquitoes, pig cycle. JE virus is transmitted to the uninfected pigs by mosquito bite. The pigs suffer from a clinically in-apparent infection. As the mosquito's density increases there is spillover of the infection to human beings following a mosquito bite. Man is the dead end host because of transient and low level of viremia in cattle. The wading birds such as egret and herons are alternate amplifying host.

\section{Pathogenesis}

JEV is introduced into human body by mosquito's bite. The virus multiplies in the epithelial cell and rarely spread to the lymph nodes. The virus spread by primary viremia and further multiplies in the reticuloendothelial system and produces secondary viremia which deposits the virus in target organ such as brain, kidney and liver. Within 7 days of primary JEV infection a rapid IgM response occurs and by one-month IgM response decreases and $\operatorname{IgG}$ antibodies appear.

\section{Clinical Presentation}

The clinical picture of JE is derived into a prodromal period of 2-5 days, encephalitis stage of 1-3 weeks and convalescent phase of weeks to several months. The Patients present with fever and behavioral abnormality or altered sensorium which ranges from drowsiness to coma, 
decerebration and decortication may also be present.

\section{Seizure}

In a study on 148 patients with AES Seizure were present in $42.6 \%$; commonest being herpes simplex encephalitis (75\%) followed by JE in $54 \%$. The predictors of seizure were Glasgow coma scale (GCS) score cortical involvement on MRI. Seizure were related to 3month outcome but not to mortality. CNS infection are commonest cause of status epilepticus (SE) in India and 30\% patients with SE are due to viral encephalitis which refractory to anticonvulsants $(7,8)$.

\section{Anterior horn cell involvement}

Focal neurological signs appear as general condition of encephalitis patients improves and consciousness returns. The focal signs include hemiplegia, quadriplegia, cerebellar signs, lower motor neurons sign; cranial nerve palsy is in rare JE. Lower motor neurons sign manifesting with focal weakness, loss of tendon reflexes have been reported in acute stage of JE (9). In JE the muscle wasting is patchy and involves a few muscles of lower limbs or noncontiguous muscle or diffuse involvement of all 4 limbs. Concentric needle EMG after 3-4 weeks of encephalitis reveals profuse fibrillates in the affected muscle. The needle EMG after 3month reveals, profound reduction or disappearance of fibrillation in the affected muscle. Motor and sensory nerve conduction are normal though motor action potential be reduced or un-recordable in some patients. The neurophysiological findings have been reported to be consistent with anterior horn cell involvement (9). There results were further confirmed in a larger study on 65 patients 23 of whom had anterior horn cell involvement but anterior horn cell involvement was not related to 3-month outcome (10).

\section{Movement disorder}

In the acute stage of JE, a wide variety of movement disorder such parkinsonian features dystonia chorea etc. have been reported $(11,12)$. In a study on 209 patients with encephalitis, 74 developed movement disorder $67.6 \%$ of these patients had JE, $51.2 \%$ had nonspecific encephalitis and $11.3 \%$ had dengue encephalitis (13). Three types of movement disorder have been reported in JE; transient form of Parkinsonism, dystonia and miscellaneous movement disorders. In a study on 50 patients 35 had movement disorder; 16 had Parkinsonism features and 19 had dystonia, in addition to Parkinsonian features. The prognosis of patients with only Parkinsonian features was better than those with additional dystonia (14). Dystonia in JE involves both the axial and limb muscle and commonly of fixed type resulting in retrocollis, opisthotonus mouth open and limb dystonia (Fig. 1). Occasionally the dystonia in JE may be

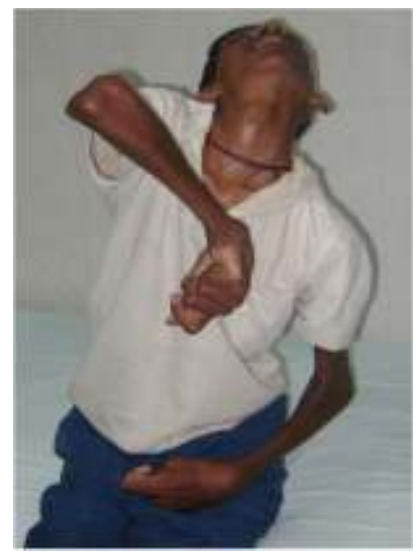

Fig 1. A 14 years old boy with JE showing severe axial and limb dystonia. 
very severe simulating status dystonicus. The dystonic spasms occur 10-30 times per day, each lasting for 10-30 minute with autonomic disturbance, swallowing, feeding and respiratory difficulty, fever and high serum CK level. These attacks are resistant to various to antidystonia therapies and regress over several months $(15,16)$. Dystonia is more common in children compared to adult and is associated with poor outcome compared to those without dystonia (17).

The movement disorder in JE are attributed to common involvement of thalamus basal ganglia and brainstem in MRI studies (18) and autopsy studies (19). The movement disorder appears as the patients recovers from coma. The basis of movement disorder in JE was associated with low CSF catecholamine levels. In the acute stage of encephalitis CSF noradrenaline, DOPEC, 5HT, HVA levels were significantly lower compared to controls. NE levels significantly correlated with dystonia and thalamic lesions (20).

\section{Radiological Findings}

CT scans findings in JE were first reported as low density areas in thalamus basal ganglia and substantia nigra (21). The changes in thalamus, insula, hippocampus and putamen are reported (22). We reported the diagnostic significance of radiological findings in the endemic area. The CT changes appeared within a week and if CT scan was normal the changes were evident on MRI as T2 hyper intensity in thalamus, basal ganglia and brainstem ( Fig. 2) (23). On MRI study of JE patient, thalamus is involved in 94\%, basal ganglia in $35 \%$ and midbrain 58\% (24). Additional temporal lobe involvement in JE has been reported $17.7 \%$ patients (25).

The involvement of thalamus and its role in movement disorders: Dystonia and parkinsonian features are the commonest movement disorder in JE and occur in isolation in combination $(51.3 \%)$. This is may be due to widespread MRI changes in thalamus basal ganglia and substantia nigra. Cortical activation disinhibits the striatum which is somatotopically organized and striatal $\mathrm{m}$ neurons and its collaterals to basal ganglia surround and inhibit the striatal neurons. Damage to the circuit involving thalamus basal ganglia, stratum and brain stem can result in different hyperkinetic or hypokinetic movement disorders (13). The parkinsonism features in JE may be due to the involvement of thalamus. The important role of thalamus in releasing basal ganglia inputs to

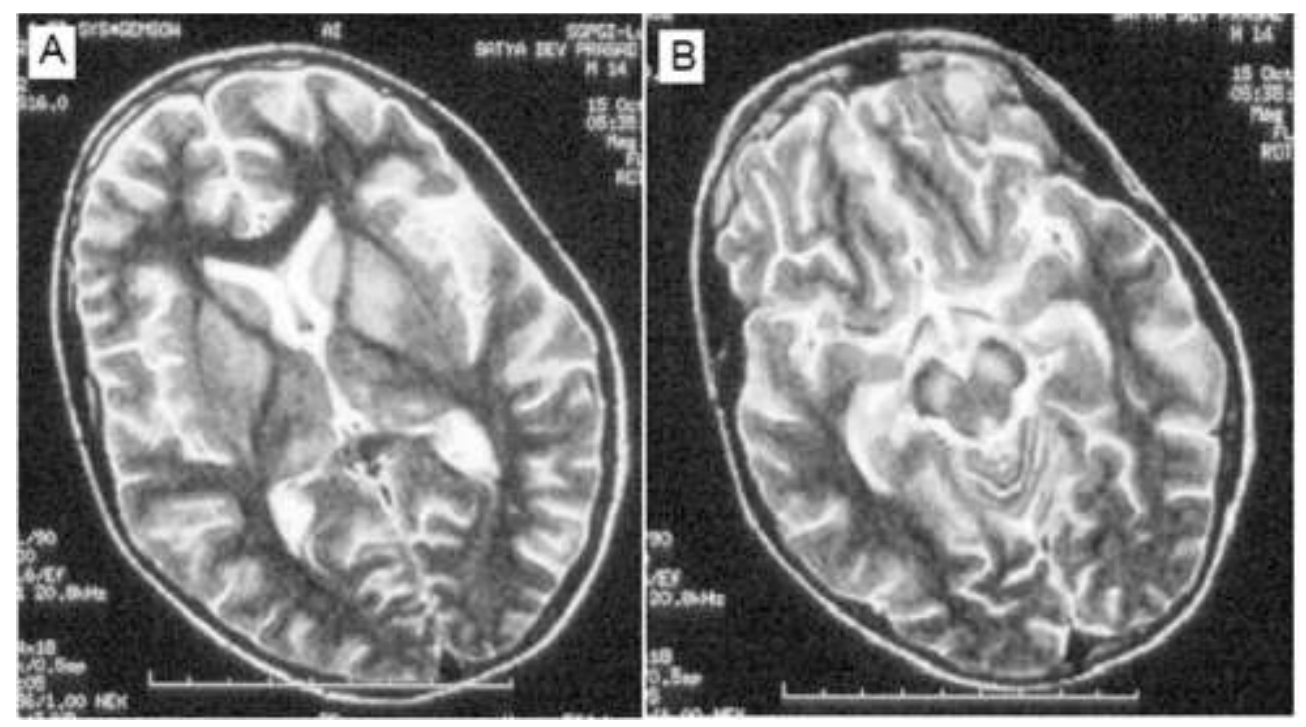

Fig.2 : Cranial MRI, T2 sequence axial section showing hyper intensity in thalamus and basal ganglia (A) and in midbrain (B) 
motor cortex is well known. On SPECT study thalamus and frontal hypo perfusion was reported. These changes support the above mentioned mechanism of movement disorders in JE. The movement disorder irrespective of its severity regress over a period of time.

The basis of movement disorders was investigated in experimental rat model as well (26). The level of nor epinephrine, dopamine, 3,4-dihydroxyphenlyacetic acid, homovalinic acid and serotonin were significantly decreased in thalamus midbrain, corpus stratum and frontal cortex. There was no significant recovery in catecholamine levels in behavioral and locomotor activities within 20 days of inoculation of JEV intracerebrally, however, histopathological changes revealed mild reduction in cell damage by $20 \mathrm{dpi}$. These result supports the clinical studies and the CSF catecholamine levels in JE patients (26).

\section{Sequelae and Prognosis}

About $20-40 \%$ patients with JE die in the acute stage (27). $50 \%$ of surviving patients have serious, behavioral abnormality, focal weakness, seizure $(20 \%)$ and a variety of movement disorder. The extent of MRI findings is not related to the outcome. The experimental studies on JE revealed impaired learning and memory function for 10-33 dpi. Improvement of memory learning was associated with reduction in expression of CHAT, HQNB CHR M2. These changes were followed by recovery after $30 \mathrm{dpi}$ (28). These results are consistent with cognitive and behavioral impairment in JE and provide some information about its underlying mechanisms.

\section{Differential diagnosis}

The patients with JE needs to be differentiated for other causes of encephalitis but its differentiation from herpes simplex encephalitis is most important, dengue, malaria and scrub typhus are also important because of different therapeutic approaches. The differentiation between HSE and JE is crucial in the early stage. Behavioral abnormalities, seizures and status epilepticus were more common in HSE whereas focal reflex loss, and movement disorder were more common in JE. Behavioral abnormality and seizure are known features of HSE because of selective involvement of frontal, temporal limbic cortex (29-32) which is highly epileptogenic and has important role in modulating memory and behavior. Temporal lobe involvement in JE is less common and reported in 19\% patients (25). Seizure in acute stage HSE have been reported in $40-60 \%(8,33)$ and JE $7 \%$ to $69 \%(17,34)$. JE is an meningoencephalomyelitis and anterior horn cell involvement occur in JE in autopsy studies $(35,36)$. A polio like illness in JE has been reported from Vietnam (37). Anterior horn cell less in JE may manifest with focal reflex less, weakness and wasting. MRI is invaluable in differentiating JE from HSE. Involvement of fronto- temporal cortex in HSE and of thalamus, brainstem and basal ganglia suggest in $\mathrm{JE}$ is highly suggestive.

There are number of other viral and nonviral condition which can which can simulate AES and have to be considered for rational and cost effective approach.

The diagnosis of AES varies according to the geographical region, and season because of differences in the prevalence of organism and their vectors. Geographically restricted encephalitis is mostly arthropod borne. The common cause of encephalitis in India are JE, dengue chikungunya, West Nile. However, malaria and scrub typhus are also common non viral infections in India. The patients with AES are generally evaluated with serum, CSF test, MRI, EEG. Those patients also receive empirical acyclovir and antibiotic therapy, taking care of most of the treatable etiologies.

A syndrome approach to AES has been suggested by us in which the patients are categorized into pure neurological or those with systemic manifestation such as rash, 
thrombocytopenia, myalgia or raised serum creatinine kinase, liver or kidney dysfunction, lymphadenopathy etc. JE and HSE are prototype of pure neurological encephalitis group. In a study rash and myalgia separated these major groups of pure neurologic and systemic AES with high sensitivity and specificity. In pure neurological AES MRI helps in differentiating JE for HSE (38). In systemic AES group, dengue, scrub typhus, chickenguniya and malaria should be considered and treated with appropriate therapy: doxycycline for ST and artesonate for malaria and ceftriaxone for leptospira. In the systemic AES group MRI, PCR for HSE and JE IgM ELISA and acyclovir therapy may not be necessary. Using the above mentioned syndromic approach in diagnosis and treatment of AES can be substantially reduced (39).

\section{Way Forward}

The spectrum of encephalitis syndrome in dynamic, Vectors control and, environmental modification and vaccination would result in reduction of some causes of AES and their space would be occupied by other etiologies. Therefore, the spectrum of encephalitis may be changing. Autoimmune encephalitis triggered by infections may be recognized more commonly and pose therapeutic challenge. The syndromic approach in AES need to be consolidated and modified in different geographical regions as per local prevalence of pathogens for cost effective rational management of AES.

\section{References}

1. Solomon T, Vaughn DW (2002). Pathogenesis and clinical features of Japanese encephalitis and West Nile virus infections. Curr Top Microbiol Immunol 267: 171-194.

2. Lee HW, Cho PY, Moon SU, et al (2015). Current situation of scrub typhus in South Korea from 2001-2013. Parasit Vectors $8: 238$.
3. Smithburn KC, Kerr JA, Gatne PB (1954). Neutralizing antibodies against certain viruses in the sera of residents of India. $J$ Immunol 72(4): 248-257.

4. Chakravarty SK, Sarkar JK, Chakravarty MS (1975). The first epidemic of Japanese encephalitis studied in India-virological studies. Indian J Med Res 63:77-82.

5. Nag D, Misra UK, Shukla R, et al (1981). Some observations on Japanese encephalitis in Uttar Pradesh. $J$ Assoc Phys India 2: 293-298.

6. Mohan Rao CVR, Benerjee K, Mandke $\mathrm{VB}$, et al (1980). Investigations of the 1978 epidemic of encephalitis in Asansol, West Bengal and Dhanbad-Bihar. J Assoc Phys India 28: 1-9.

7. Kalita J, Nair PP, Misra UK (2008). Status epilepticus in encephalitis: a study of clinical findings, magnetic resonance imaging, and response to antiepileptic drugs. JNeurovirol 14: 412-417.

8. Misra UK, Tan CT, Kalita J, et al (2008). Viral encephalitis and epilepsy. Epilepsia 49 Suppl 6: 13-18.

9. Misra UK, Kalita J (1997). Anterior horn cells are also involved in Japanese encephalitis. Acta Neurol Scand 96(2): 114-117.

10. Kalita J, Misra UK ( $\left.\begin{array}{llll}2 & 0 & 0 & 2\end{array}\right)$. Neurophysiological changes in Japanese encephalitis. Neurol India 50: 262-266.

11. Gourie-Devi M, Ravi V, Shankar SK (1995). Japanese encephalitis: an overview. In : Recent Advances in Tropical Neurology. Clifford R, ed. Amsterdam: Elsevier Science BV, 217235.

12. Prasad SR, George S, Gupta NP (1982). Studies on an outbreak of Japanese encephalitis in Kolar district, Karnataka 
state in 1977-78. Indian J Med Res 75:16.

13. Misra UK, Kalita J (2010). Spectrum of movement disorders in encephalitis. $J$ Neurol 257:2052-2058.

14. Misra UK, Kalita J (2002). Prognosis of Japanese encephalitis patients with dystonia compared to those with parkinsonian features only. Postgrad Med J78:238-241.

15. Kalita J, Misra UK (2000). Markedly severe dystonia in Japanese encephalitis. MovDisord 15:1168-1172.

16. Kalita J, Misra UK, Pradhan PK (2011). Oromandibular dystonia in encephalitis. $J$ NeurolSci 304(1-2): 107-110.

17. Kalita Misra, Pandey S, Dhole TN (2003). A comparison of clinical and radiological findings in adults and children with Japanese encephalitis. Arch Neurol 60(12): $1760-1764$

18. Kalita J, Misra UK (2000). The substantia nigra is also involved in Japanese encephalitis. AJNR Am J Neuroradiol 21(10): 1978-1980.

19. Zimmerman HM (1946). The pathology of Japanese B encephalitis. Am J Pathol 22:965-991.

20. Kalita J, Kumar S, Vijaykumar K, Palit G, Misra UK (2007). A study of CSF catecholamine and its metabolites in acute and convalescent period of encephalitis. $J$ Neurol Sci 252: 62-66.

21. Toyomasu T, Nakashima K, Matsumoto T, Shida K (1982). CT findings in a case of Japanese encephalitis. Rinsho Shinkeigaku 22(10): 891-895.
22. Kumar S, Misra UK, Kalita J, Salwani V, Gupta RK, Gujral R (1997). MRI in Japanese encephalitis. Neuroradiology 39: $180-184$.

23. Misra UK, Kalita J, Jain SK, Mathur A ( 1994 ). Radiologic al and neurophysiological changes in Japanese encephalitis. J Neurol Neurosurg Psych 57(12): 1484-1487.

24. Kalita J, Misra UK (2000). Comparison of CT scan and MRI findings in the diagnosis of Japanese encephalitis. J Neurol Sci 174(1): 3-8.

25. Handique SK, Das RR, Barman K, et al (2006). Temporal lobe involvement in Japanese encephalitis: problems in differential diagnosis. AJNR Am $J$ Neuroradiol 27(5):1027-1031.

26. Misra UK, Kalita J, Jain SK, Mathur A (1994). Radiological and neurophysiological changes in Japanese encephalitis. J Neurol Neurosurg Psych 57(12): 1484-1487.

27. Misra UK, Kalita J (2010). Overview: Japanese encephalitis. Prog Neurobiol 91:108-120.

28. Chauhan PS, Khanna VK, Kalita J, Misra UK (2017). Japanese encephalitis virus infection results in transient dysfunction of memory learning and cholinesterase inhibition. MolNeurobiol 54: 4705-4715.

29. Damasio AR, Van Hoesen GW (1985). The limbic system and the localisation of herpes simplex encephalitis. J Neurol Neurosurg Psych 48: 297-301.

30. McCabe K, Tyler K, Tanabe J (2003). Diffusion-weighted MRI abnormalities as a clue to the diagnosis of herpes simplex encephalitis. Neurology 61(7):1015-1016. 
31. Misra UK, Kalita J, Phadke RV, et al (2010). Usefulness of various MRI sequences in the diagnosis of viral encephalitis. Acta Trop 116(3):206-211.

32. Sili U, Kaya A, Mert A; HSVEncephalitis Study Group (2014). Herpes simplex virus encephalitis: clinical manifestations, diagnosis and outcome in 106 adult patients. J Clin Virol 60: 112-118.

33. McGrath N, Anderson NE, Croxson MC, Powell KF (1997). Herpes simplex encephalitis treated with acyclovir: diagnosis and long term outcome. $J$ Neurol Neurosurg Psych 63(3): 321-326.

34. Chen YJ, Fang PC, Chow JC (2006). Clinical characteristics and prognostic factors of postencephalitic epilepsy in children. J Child Neurol 21(12): 10471051.

35. Chatterjee A Banerjee K (1975). Epidemiological studies on the encephalitis epidemic in Bankura. Indian JMedRes 63:1164-1177.

36. Wadia NH, Khadilkar SV (2015). Neurological Practice: An Indian Perspective-E-Book, 2nd edn. Elsevier India.

37. Solomon T, Kneen R, Dung NM, et al (1998). Poliomyelitis-like illness due to Japanese encephalitis virus. Lancet 351(9109): 1094-1097.

38. Kalita J, Misra UK, Mani VE, Bhoi SK (2016). Can we differentiate between herpes simplex encephalitis and Japanese encephalitis? JNeurol Sci 366:110-115.

39. Misra UK, Mani VE, Kalita J (2017). A Cost-Effective Approach to the Diagnosis and Management of Acute Infectious Encephalitis. Eur Neurol 77(1-2): 66-74. 